MAGNETOHYDRODYNAMICS Vol. 54 (2018), No. 1-2, pp. 79-83

DOI: $10.22364 / \mathrm{mhd} .54 .1-2.14$

\title{
THE FORM OF THE MAGNETIC FLUID DROP IN A UNIFORM HARMONIC ELECTRIC FIELD WITH THE CONCENTRATION OF SURFACE CHARGE TRANSPORTATIONS
}

\author{
D. Kvasov ${ }^{1}$, V. Naletova ${ }^{1,2}$ \\ 1 Lomonosov Moscow State University, 119991 Moscow, Russian Federation, \\ ${ }^{2}$ Institute of Mechanics, 119192 Moscow, Russian Federation
}

A magnetic fluid drop immersed in oil and exposed to a harmonic electric field is studied theoretically. All surface charge transportations are considered. It is shown that the surface conductivity can quantitatively change the deformation, but cannot change it qualitatively.

Introduction. Deformation of liquid drops was investigated in many works [17]. The main method of investigation is the approximation by the small parameter which determines the relations between electric and capillary forces. In [1-3], the deformation was found using only the first approximation by the small parameter. In this approximation, it is impossible to take into account the convective charge transportations. In [4], the deformation was found for a steady electric field using the second approximation and thus the convective charge transportations were considered. In [5], the behavior of a drop of kerosene-based magnetic fluid immersed in the AMG-10 oil and subjected to a uniform harmonic electric field was experimentally studied. It is shown that the drop can have an oblate shape instead of the predicted prolate shape. In [6,7], the drop shape was investigated under the action of a harmonic electric field using the second approximation by the small parameter. In all studies cited above, the surface conductivity was not taken into account. In [8], the surface conductivity was considered, but only for spherical drops without any deformation.

In this paper, the shape of the drop is investigated in the harmonic electric field. All surface charge transportations are considered.

1. Problem formulation. Let us consider a drop of magnetic fluid in oil. The fluids are assumed to be weakly conducting and all their properties are uniform. The presence of the electric field leads to vorticity inside and outside the drop. The indices ' $i$ ', 'e' for the fluid parameters denote them as internal and external fluids, accordingly. The index 's' denotes parameters on the surface. The coordinate system is chosen as in Fig. 1. The surface metric tensor in these coordinates has the form:

$$
g_{\theta \theta}=\left(f^{2}+f^{\prime 2}\right), g_{\phi \phi}=f^{2} \sin ^{2} \theta, g_{\theta \phi}=g_{i \phi \theta}=0, g=g_{\theta \theta} g_{\phi \phi} .
$$

We consider flows with low velocities, therefore, the Reynolds numbers $R$ are small and the Stokes approximation can be used. The dimensionless equations that describe such fluid flow take the form (the notation is common and the same as in $[7])$ :

$$
\mathbf{E}=-\nabla \phi, \Delta \phi_{k}=0, \operatorname{div} \mathbf{v}_{k}=0, \alpha \operatorname{Sh} R \frac{\partial \mathbf{v}_{k}}{\partial t}=-\nabla p_{k}+\alpha M_{k} \Delta \mathbf{v}_{k}, k \in\{i, e\} .
$$




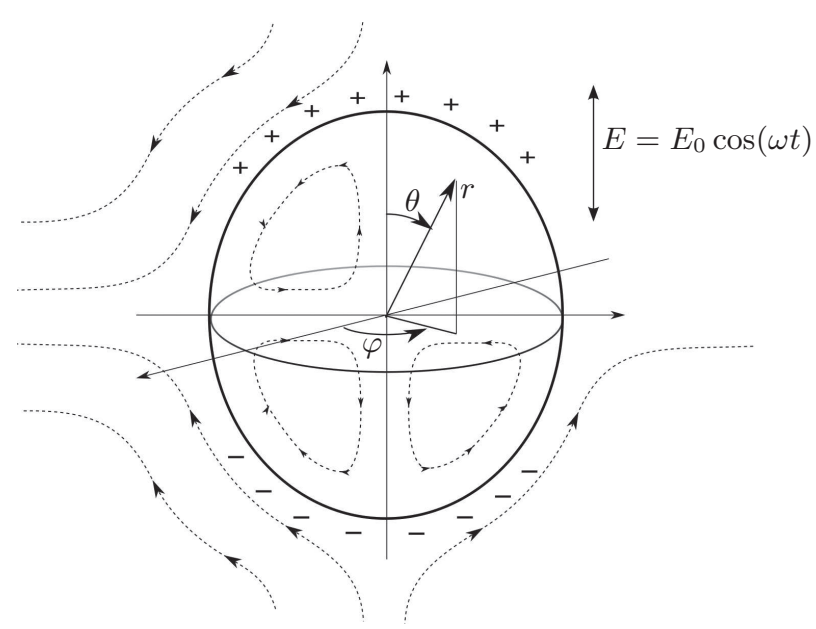

Fig. 1. Schematic presentation of the drop and streamlines.

The dimensionless boundary conditions on the surface are

$$
\begin{aligned}
& {[\phi]=0, E_{n e}-\varepsilon E_{n i}=q_{\mathrm{s}}, v_{\tau i}=v_{\tau e}, v_{n i}=v_{n e}=\operatorname{Sh} \sqrt{1+f^{\prime 2} / f^{2}} \frac{\partial f}{\partial t},} \\
& \left(E_{n e}-\Lambda E_{n i}\right)+\frac{\partial \Lambda_{\mathrm{s}} E_{\tau} \sqrt{g_{\phi \phi}}}{\sqrt{g} \partial \theta}+\alpha \gamma \frac{\partial q_{\mathrm{s}} v_{\tau} \sqrt{g_{\phi \phi}}}{\sqrt{g} \partial \theta}+\Omega_{\mathrm{e}} \frac{\partial q_{\mathrm{s}} \sqrt{g}}{\sqrt{g} \partial t}=0, \\
& {[p] \mathbf{n}-\alpha[M \Pi]=-2 K \mathbf{n}+\alpha\left(E_{n e} \mathbf{E}_{\mathrm{e}}-\varepsilon E_{n i} \mathbf{E}_{\mathrm{i}}-\frac{1}{2}\left(E_{\mathrm{e}}^{2}-\varepsilon E_{\mathrm{i}}^{2}\right) \mathbf{n}\right) .}
\end{aligned}
$$

The dimensionless boundary conditions at infinity are $v \rightarrow 0, \phi \rightarrow-r E_{0} \cos \theta$. Here $K$ is the mean curvature, $\Pi$ is a dimensionless vector of viscous stresses. Also, the following dimensionless parameters are used:

$$
\begin{aligned}
& r^{*}=\frac{r}{r_{0}}, p^{*}=\frac{p r_{0}}{T}, v^{*}=\frac{v}{v_{c}}, E^{*}=\frac{E}{E_{0}}, \phi^{*}=\frac{\phi}{E_{0} r_{0}}, q_{\mathrm{s}}^{*}=\frac{q_{\mathrm{s}}}{\varepsilon_{\mathrm{e}} \varepsilon_{0} E_{0}}, t^{*}=t \omega, \\
& \Lambda=\frac{\lambda_{\mathrm{i}}}{\lambda_{\mathrm{e}}}, \varepsilon=\frac{\varepsilon_{\mathrm{i}}}{\varepsilon_{\mathrm{e}}}, \mathrm{M}_{\mathrm{i}}=\frac{\mu_{\mathrm{i}}}{\mu_{\mathrm{i}}+\mu_{\mathrm{e}}}, \mathrm{M}_{\mathrm{e}}=\frac{\mu_{\mathrm{e}}}{\mu_{\mathrm{i}}+\mu_{\mathrm{e}}}, \Omega_{\mathrm{i}}=\frac{\varepsilon_{0} \varepsilon_{\mathrm{i}}}{\lambda_{\mathrm{i}}} \omega, \Omega_{\mathrm{e}}=\frac{\varepsilon_{0} \varepsilon_{\mathrm{e}}}{\lambda_{\mathrm{e}}} \omega, \\
& \Lambda_{\mathrm{s}}=\frac{\lambda_{\mathrm{s}}}{\lambda_{\mathrm{e}} r_{0}}, \alpha=\frac{\varepsilon_{\mathrm{e}} \varepsilon_{0} E_{0}^{2} r_{0}}{T}, \gamma=\frac{\varepsilon_{\mathrm{e}} \varepsilon_{0} T}{\lambda_{\mathrm{e}}\left(\mu_{\mathrm{e}}+\mu_{\mathrm{i}}\right) r_{0}}, \mathrm{R}=\frac{\rho v_{c} r_{0}}{\mu_{\mathrm{e}}+\mu_{\mathrm{i}}}, \mathrm{Sh}=\frac{r_{0} \omega}{v_{c}}
\end{aligned}
$$

where $T$ is the interfacial tension, $v_{c}=\varepsilon_{\mathrm{e}} \varepsilon_{0} E_{0}^{2} r_{0} /\left(\mu_{\mathrm{e}}+\mu_{\mathrm{i}}\right)$ is a characteristic velocity.

2. Solution. It is assumed that the parameter $\alpha$ is small $(\alpha \ll 1)$, and for the solution it is found in the form of expansion by powers of $\alpha$ :

$$
\begin{aligned}
f(\theta, t) & =f^{(0)}(\theta, t)+\alpha f^{(1)}(\theta, t)+\alpha^{2} f^{(2)}(\theta, t)+\ldots, \\
\Phi(r, \theta, t) & =\Phi^{(0)}(r, \theta, t)+\alpha \Phi^{(1)}(r, \theta, t)+\alpha^{2} \Phi^{(2)}(r, \theta, t)+\ldots,
\end{aligned}
$$

where $\Phi$ denotes any of the variables $p_{k}, \mathbf{v}_{k}, \phi_{k}, q_{\mathrm{s}}, k \in\{i, e\}$. To characterize the drop shape, a parameter called "deformation" will be used $(D>0$ and $D<0$ correspond to the prolate and oblate shapes, respectively):

$$
D=\frac{f(\theta=0)-f(\theta=\pi / 2)}{2} .
$$


The form of the magnetic fluid drop in a uniform harmonic electric field with the...

(a)

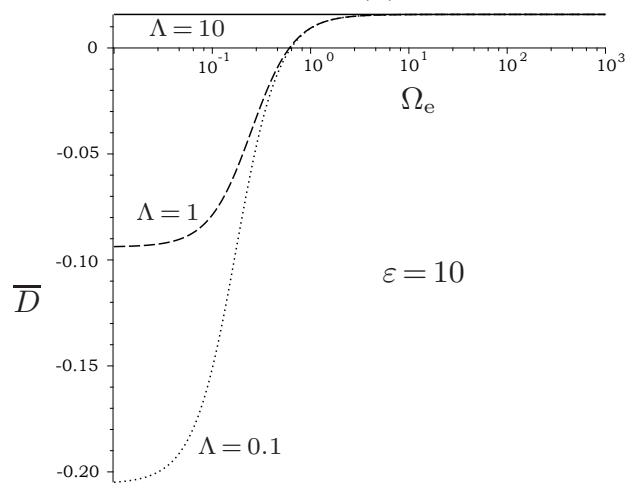

(b)

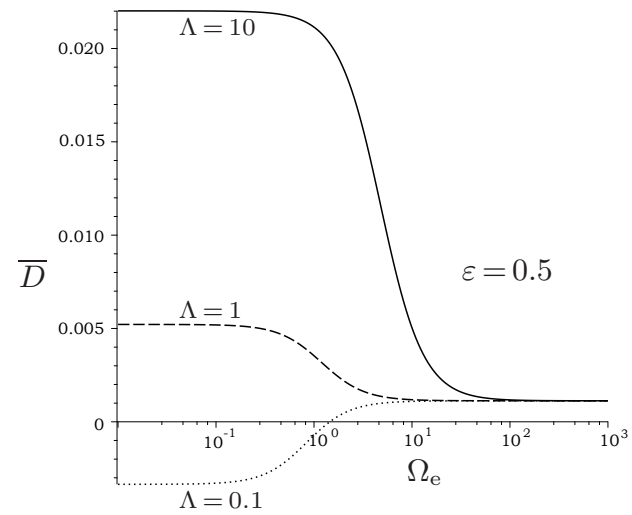

Fig. 2. Dependence of $\overline{D^{(1)}}$ on $\Omega_{\mathrm{e}}$ for $\Lambda_{\mathrm{s}}=0.05$, first approximation.

2.1. First approximation. Using this approximation, the deformation was found in $[2,3]$ without considering the surface conductivity. The solution, including the surface conductivity, for the electric potential is

$$
\begin{gathered}
\phi_{\mathrm{e}}^{(0)}=-\left(r+\frac{1-L}{(L+2) r^{2}}\right) \cos (\theta) \mathrm{e}^{i t}, \quad \phi_{\mathrm{i}}^{(0)}=-\frac{3 r}{L+2} \cos (\theta) \mathrm{e}^{i t}, \\
q_{\mathrm{s}}^{(0)}=3 \frac{L-\varepsilon}{L+2} \cos (\theta) \mathrm{e}^{i t}, \quad L=\frac{\Lambda+2 \Lambda_{\mathrm{s}}+i \Omega_{\mathrm{e}}}{1+i \Omega_{\mathrm{e}}} .
\end{gathered}
$$

By solving the hydrodynamic part of the equations it is possible to find the drop surface shape as

$$
\begin{aligned}
f^{(1)} & =2\left(X+\Re \mathrm{e}\left\{H \mathrm{e}^{2 i t}\right\}\right) P_{2}, \quad P_{2}=\left(3 \cos ^{2}(\theta)-1\right) / 2, \\
X & =\frac{9\left(\Lambda+2 \Lambda_{\mathrm{s}}-\varepsilon\right)\left(2+M_{\mathrm{i}}\right)+15\left(\left(\Lambda+2 \Lambda_{\mathrm{s}}\right)^{2}+\Omega_{\mathrm{e}}^{2}(\varepsilon-1)^{2}+1-2 \varepsilon\right)}{80\left(\left(\Lambda+2 \Lambda_{\mathrm{s}}+2\right)^{2}+\Omega_{\mathrm{e}}^{2}(\varepsilon+2)^{2}\right)} .
\end{aligned}
$$

This result, with the substitution of $\Lambda_{\mathrm{s}}=0$, is same as in [7]. The full expression for $H$ is not given because here we need only the steady part of the shape. The deformation parameter for the steady part in this approximation is

$$
\overline{D^{(1)}}=\frac{\overline{f^{(1)}}(\theta=0)-\overline{f^{(1)}}(\theta=\pi / 2)}{2} .
$$

From Eq. (8) it is easy to find that the drop is always prolate on a big enough $\Omega_{\mathrm{e}}$. The parameter $\Lambda_{\mathrm{s}}$ appears only in the sum $\Lambda+2 \Lambda_{\mathrm{s}}$ and it could be replaced by an additional conductivity of the internal fluid. This fact leads to a conclusion that the surface conductivity in the first approximation cannot make the drop less prolate. There are some plots showing the dependence of $\bar{D}$ on $\Omega_{\mathrm{e}}$ for fluids with the same $M_{\mathrm{i}}=0.01$ and $\varepsilon$ (see Fig. 2 and Fig. 3). There is an obvious inverse proportionality between the conductivity and the oblateness. So, in cases when $\varepsilon$ is big enough, the smaller $\Lambda$, the more oblate the drop becomes. There is a difference between the deformation on $\Omega_{\mathrm{e}} \rightarrow \infty$ and the deformation in the steady electric field because of the oscillatory part of deformation, which is significant at infinity. 


\section{Kvasov, V. Naletova}

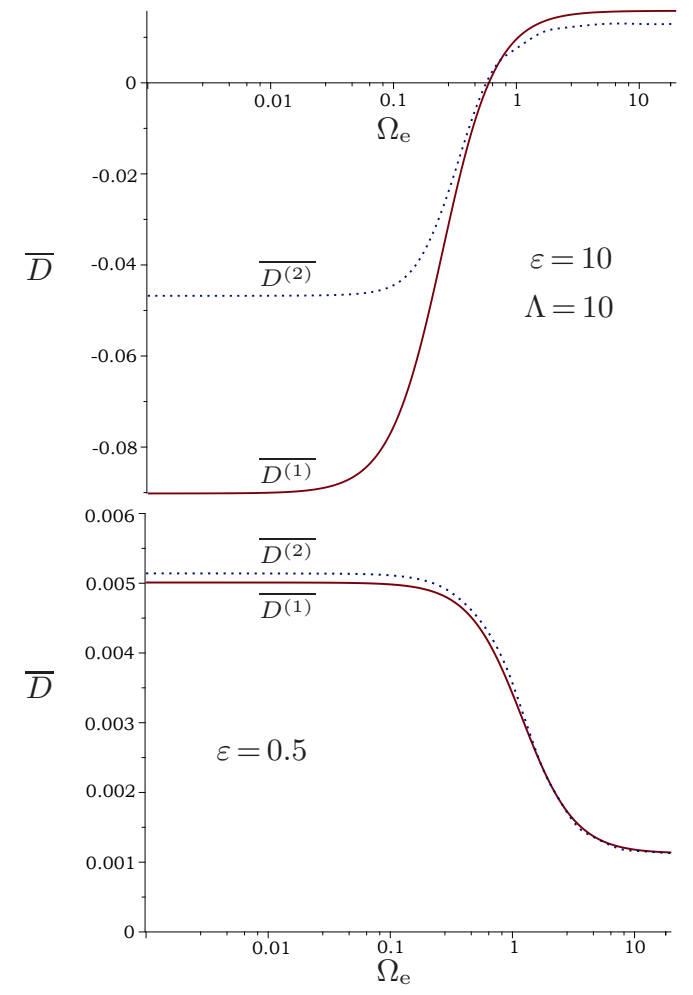

(a)

(b)

Fig. 3. Comparison of the dependences of $\overline{D^{(1)}}$ and $\overline{D^{(2)}}$ on $\Omega_{\mathrm{e}}$ for $\Lambda=0.95$ and $\Lambda_{\mathrm{s}}=0.05$, second approximation.

2.2. Second approximation. This approximation allows us to take into account the convective charge transportations which are connected with the $\gamma$ parameter. The main difficulty here is that the boundary conditions are defined on the surface which is obtained in the first approximation. To solve this problem, there is an equation for any function $A$ and small $\alpha$ :

$$
\left.A\right|_{r=1+\alpha f^{(1)}}=\left.A\right|_{r=1}+\left.\alpha f^{(1)} \frac{\partial A}{\partial r}\right|_{r=1} .
$$

The equations and the boundary conditions are mostly the same as in [7] except for the charge save equation:

$$
\begin{aligned}
{\left[E_{n}^{(1)}\right]_{\Lambda}+2\left[E_{n}^{(0)}\right]_{\Lambda} f^{(1)} } & +\frac{\partial\left(\Lambda_{\mathrm{s}}\left(E_{\tau}^{(0)} f^{(1)}+E_{\tau}^{(1)}\right)+\gamma q_{\mathrm{s}}^{(0)} v_{\tau}^{(0)}\right) \sin (\theta)}{\sin (\theta) \partial \theta} \\
& +\Omega_{\mathrm{e}}\left(\frac{\partial q_{\mathrm{s}}^{(1)}}{\partial t}+2 q_{\mathrm{s}}^{(0)} \frac{\partial f^{(1)}}{\partial t}\right)=0 .
\end{aligned}
$$

Here $[A]_{b}=A_{\mathrm{e}}-b A_{\mathrm{i}}$. All equations are solved using the same approach as in [7] and an additional steady part of the form $\overline{f^{(2)}}$ is found. Now it is possible to compare the deformation for the second approximation $\left(\overline{D^{(2)}}\right)$ with the solution for the first approximation $\left(\overline{D^{(1)}}\right)$. As shown in Figs. $3 a, 2 b$, the drop shape in the solution for the second approximaton (dots) slightly differs from that for first approximation. 
The form of the magnetic fluid drop in a uniform harmonic electric field with the...

3. Conclusion. The drop deformation is found taking into account all surface charge transportations. It is shown that in the first approximation, the presence of the surface conductivity could be replaced by the additional conductivity of the internal fluid. So, in the first approximation, the consideration of the surface conductivity could only increase the elongation of the drop. The solution for the second approximation is much more complex, but it is shown that the consideration of the surface conductivity does not make a big contribution to the deformation sign. Moreover, the surface conductivity, as a rule, tends to enlarge the elongation and reduce the oblateness of the drop. So this model does not explain the experimental data from [5].

\section{References}

[1] J.R. Melcher, G.I. TAYLOR. Electrohydrodynamics: a review of the role of interfacial shear stresses. Annual Rev. Fluid Mech., vol. 1 (1969), pp. 111-146.

[2] S. Torza, R.G. Cox, And S.G. MAson. Electrohydrodynamic deformation and burst of liquid drops. Phil. Trans. R. Soc. Lond., A 269 (1971), no. 1198, pp. $295-319$.

[3] C. Sozou. Electrohydrodynamics of a liquid drop: the time-dependent problem. Proc. R. Soc. London, A 331 (1972), p. 263.

[4] V.Ya. Shkadov, A.A. Shutov. Drop and bubble deformation in an electric field. Fluid Dynamics, vol. 37 (2002), no. 5, pp. 713-724.

[5] E.S. Tkacheva, A.R. Zakinyan. Dynamics of forms of magnetic and not magnetic drops of magneto-dielectric emulsions in magnetic and electric fields. St. Petersburg State Polytechnical University Journal, no. 4 (2009), pp. 76-82. (in Russian).

[6] Yu. Dikanskit, D.I. Kvasov, V.A. Naletova, E.S. Tkacheva. Deformation of magnetic liquid drop in a harmonic elecrtic field. In: IV All-Russian Scientific Conference on Physical and Chemical and Applied Problems of Magnetic Disperse Nanosystemss (Stavropol, 16-19 September 2013), pp. 58-64 (in Russian).

[7] D.I. Kvasov. Dielectric liquid drop in a harmonic electric field. Fluid Dynamics, vol. 51 (2016), np. 2, pp. 110-114.

[8] A.N. Tyatyushkin. Effect of surface electric current on the electrohydrodynamic flow inside and outside a spherical drop. Fluid Dynamics, vol. 52 (2017), no. 2, pp. 178-188.

Received 27.12.2017 